\title{
IV Foro Científico Internacional «Entre Dos Orillas» Migraciones: Causas y Efectos
}

\section{International Scientific Forum «Between Two Banks» Migrations: Causes and Effects \\ Trabajo de observación participante}

Ivonne Teresa Valencia León ${ }^{a}$

en Laayune, Reino de Marruecos, 4 al 7 de julio de 2019

Laayoune, Marruecos. La Universidad Mohamed V de Rabat (Facultad de Letras y Ciencias Humanas, Derecho, Economía y Ciencias Sociales) y Alter Forum Internacional, con la colaboración del Centro de Investigación Hlcr y el Laboratorio de Investigación Lt2, organizaron la cuarta edición del Foro Científico Internacional «Entre dos orillas» Migraciones: causas y efectos. Con el objetivo de ayudar en la difusión del acuerdo del Pacto Mundial de la Migración ${ }^{1}$-impulsado por el Reino de Marruecos junto a la ONU y desarrollado en la ciudad de Marrakech el 10 y 11 de diciembre de 2018- nos reunimos en Laayoune, ciudad construida en un desierto, diversos docentes investigadores provenientes de universidades de 11 países, siendo representantes de diversas culturas, para discutir sobre las problemáticas que se han generado a propósito de la migración humana por el mundo. En las siguientes líneas, vamos a presentar la descripción de las principales actividades desarrolladas y del trabajo de observación participante llevado a cabo entre los días 4 y 7 de julio, y hasta el 10 con actividades culturales, de relaciones internacionales y de un poco de turismo; las mismas que se presentarán teniendo en cuenta los siguientes criterios: ponencias internacionales, organización del evento, idiomas, espiritualidad religiosa, gastronomía árabe, estilo de vida y el desierto de Sahara.

\section{Sobre las ponencias internacionales}

En torno al movimiento migratorio de grandes cantidades de personas por el mundo, se planteó la idea de reflexionar sobre las diferentes causas y consecuencias, tanto para los propios migrantes como para los países de origen, los países de tránsito y para los países de residencia/permanencia/destino migratorio. La coordinación general del evento estuvo a cargo del Sr. Bachir Edkhil, (presidente de Alter Forum Internacional) con el apoyo del profesor Mustapha Ouzir (Director del Departamento de Estudios Hispánicos, Universidad de Mohamed V de Rabat).

El trabajo académico estuvo distribuido en seis sesiones de trabajo, teniendo en cuenta cuatro ejes temáticos relacionados con la migración: conflictos, derechos humanos, desarrollo y el medio ambiente, y la integración.

a Socióloga peruana. Docente de la Universidad Nacional Mayor de San Marcos. Invitada a participar como expositora en el Foro Internacional «Entre dos orillas» Migraciones: causas y efectos. Laayoune, Reino de Marruecos.

1 Este acuerdo también lleva por nombre «Pacto Mundial para la Migración Segura, Ordenada y Regular». Para mayor información, visitar: https://www.un.org/en/conf/migration/ 
Jueves 4 de julio. Recepción de las delegaciones internacionales. Cena de bienvenida y presentación general de la reunión a cargo del comité organizador.

Viernes 5 de julio. Inauguración. Asistencia de representantes de las entidades de Estado: el Ministerio de la Sociedad Civil, el Ministerio de Comunicación, la Gobernación y representantes de diversas facultades de la Universidad Mohamed V de Rabat.

Primera sesión. Migración y conflictos, dirigida por el profesor Mustapha Ouzir, contó con la participación del Dr. Abdelaziz Laaroussi de la Universidad Mohamed V de Rabat (Marruecos), quien presentó la ponencia «Migration á la lumière de la pratique des conventions internationales». El Dr. Gabriel Restrepo (sociólogo y escritor colombiano) presentó una interesante ponencia a la que tituló "Ires y venires, migraciones», quien planteó la idea de que en realidad todos provenimos del África, por lo que hizo mención al pasito pa adelante, un pasito pa atrás. Por su parte, la mexicana Dra. Julieta Espín Ocampo, de la Universidad Europea de Madrid (España), describió la situación problemática en Palestina, titulando a su ponencia: «Migración y conflicto en Palestina». En la misma línea, la Dra. Karim Chihi, profesora de la Universidad Jean Moulin, Lyon III (Francia), presentó su trabajo titulado «Migración y conflicto en Siria». Siguiendo con la misma temática, el Sr. Jamal Mechbal presentó el libro «Marruecos y su Sahara Occidental». Al final de la primera sesión, la colombiana Clara Riveros presentó el libro Por el desierto del Sáhara con una politóloga colombiana y un ex diplomático marroquí.

Segunda sesión. Migración y derechos humanos, dirigida por la profesora Ivonne Valencia León, contó con la participación de la Dra. Claudia Barona Castañeda, profesora de la Universidad de las Américas, Puebla (México), quien nos presentó su ponencia titulada «Las caravanas centroamericanas», donde puso en evidencia la realidad migratoria de los integrantes de las caravanas de guatemaltecos y hondureños durante su paso por México. Luego, el Dr. Mohamed Badine El Yattioui, de nacionalidad francesa pero de origen marroquí, profesor de la Universidad de las Américas, Puebla (México), presentó la ponencia «Trump y el muro con México», con la cual puso en evidencia que la idea de muro no tiene en cuenta la cuestión humanitaria y que todavía hay mucho por discutir. Finalmente, el gran escritor colombiano, Dr. Luis Fayad, presentó una reflexión acerca de «La nueva población de Europa».

Sábado 6 de julio. Tercera sesión. Migración, medio ambiente y desarrollo, dirigida por la profesora Claudia Barona, contó con la participación de la Dra. Celeste Cedillo González, profesora de la Universidad de las Américas, Puebla (México), quien presentó la ponencia titulada «Debate conceptual: ¿el medio ambiente impacta la migración y/o la migración impacta el medio Ambiente?». Luego, el Dr. Yusef Nava, miembro de Alter Forum, presentó una interesante reflexión sobre la relación entre la migración y la salud en su ponencia titulada «Migraciones y salud global: retos y oportunidades». El Dr. Pablo Neder, de nacionalidad argentina pero de origen árabe, presentó la ponencia «Los programas y organizaciones internacionales: PNUD/OIM». Más tarde, la Dra. Ivonne Valencia León, profesora de la Universidad Nacional Mayor de San Marcos (Perú), presentó la realidad problemática migratoria peruana con su ponencia titulada «Perú: país de inmigrantes y de emigrantes. Los transmigrantes del Siglo XXI». Siendo una cultura absolutamente diversa, tuvimos que presentar -en líneas generales- el proceso histórico de la emigración de peruanos al exterior y de la inmigración extranjera residente en el país, especialmente, el actual fenómeno migratorio venezolano.

Cuarta sesión. Migraciones y Desarrollo, dirigida por Pablo Neder, contó con la participación de la Dra. Fauzaya Talhaoui, diputada del Parlamento Federal de Bélgica, quien presentó una ponencia titulada «La réintégration des réfugiés et migrants dans leur pays d'origine par moyen de formation et entreprenariat. Cas Green Goat, star up incubateur dans l'économie bio basée», interesante trabajo sobre la reintegración de los inmigrantes extranjeros, quienes participan de proyectos/emprendimientos sociales. 
Luego, el estudiante de doctorado, Nicolas Klingelschmitt, de la Universidad Québec de Montréal (Canadá), presentó la ponencia, «Los programas regionales: Fondo Fecundario de la UE para África». Al finalizar la jornada, se proyectó un documental sobre la música y la migración en países europeos.

Domingo 7 de julio. Quinta sesión. Migraciones y desarrollo (continuación), dirigido por el profesor Mohamed Badine El Yattioui, contó con la participación de Vanessa Gutiérrez, ingeniera consultora en tecnología, de origen venezolano y residente en Argentina, quien presentó la ponencia «Inmigración, tecnología y emprendimiento» y mostró cómo el uso de las tecnologías, a través de las redes sociales, permite desarrollarse a los extranjeros en Argentina. Así, comentó el caso de los youtubers e influencers migrantes y el éxito que alcanzaron. Más tarde, el Dr. Johan Wets, profesor de la Université Catholique de Louvain (Bélgica), mediante un vídeo grabado, presentó la ponencia «L'inéviabilité des migrantions internationales: un défi mondial pour l'avenir». En la última sesión, la Dra. Ana Silva, profesora de la Universidad de Granada (España), presentó la ponencia titulada «Migración y Género», llamando la atención sobre una problemática que ya tienen algún tiempo dedicado en las mesas de discusión, sin embargo, expone la necesidad de trabajar y mejorar las gestiones en cuanto la protección de las mujeres migrantes. Luego, participaron docentes y estudiantes de posgrado que abordaron el tema de la migración de la comunidad marroquí en España. Así, el Dr. Zakaria Boudhim, de la Universidad Mohamed V de Rabat (Marruecos), se refirió a «La contribución de los emigrantes marroquíes en el desarrollo económico de Italia»; y Najmi Abdelkhalak, de origen marroquí y estudiante de doctorado de la Universidad Autónoma de Madrid (España), abordó el tema «La situación de los estudiantes marroquíes en España». En la misma línea, Fatma Hemdani, de la Universidad Hassan II de Casablanca (Marruecos), presentó los resultados de su investigación acerca de la «Integración de la mujer emigrante marroqui en España».

La última etapa del Foro estuvo dedicada a presentaciones literarias. Participaron el Dr. Gabriel Restrepo y Bachir Edkhil, quienes presentaron el libro Marruecos, Rosa de los vientos. Luego, Sebastián Álvarez Posada, profesor de la Universidad de Medellín (Colombia) y Mohamed Badine El Yattioui, profesor de la Universidad de las Américas, Puebla (México), presentaron el libro El Islam desde una perspectiva geopolítica.

El Foro culminó con una discusión y la firma del «Acta fundacional del proyecto de cooperación Sur-Sur. Sembrar la Paz desde las raíces», dirigida por los fundadores de la Nueva Ruta Libertadora por la Paz y la Educación, Bachir Edkhil y Gabriel Restrepo. Participaron Mustapha Ouzir, Claudia Barona Castañeda, Mohammed Badine El Yattioui, J.M. Lizundia y Yusef Nava. Los invitados internacionales nos sumamos con nuestras firmas, indicando la institución a la que representamos. Al terminar, se tomaron las fotografías como evidencia del trabajo realizado.

\section{Sobre los organizadores}

La organización de evento estuvo a cargo del politólogo y poeta marroquí Bachir Edkhil, presidente de Alter Forum Internacional, con el apoyo de Mustapha Ouzir, profesor de la Universidad Mohamed V de Rabat. La acogida de los invitados internacionales, estuvo muy bien planteada por parte de todo el equipo de trabajo, especialmente de los docentes y alumnos y de la Universidad de Mohamed V de Rabat. En las siguiente líneas anotamos los nombres de todas las personas involucradas: Jamal Eddine El Hani (Decano de la Facultad de Letras y Ciencias Humanas), Mohammed Zakaria Abouddahab (Decano de la Facultad de Derecho, Economía y Ciencias Sociales), Yamina El Kirat (Vicedecana de la Facultad de Letras y Ciencias Humanas), Abdelaziz Laaroussi (Vicedecano de la Facultad de Derecho, Economía y Ciencias Sociales), Youssef Nava (Alter Forum Internacional), Zakaria Boudhim (Coordinador del Máster de traducción), Rachis Morsli (Jefe del Departamento de Estudios 
Germánicos), Redouan Hasban (Departamento de Estudios Germánicos, Claudia Barona Castañeda (Universidad de las Américas Puebla, México), Mohamed Badine El Yattioui (Universidad de las Américas Puebla, México), Clara Riveros (Observatorio CPLATAM, Colombia), Pablo Eduardo Neder (Instituto Latinoamericano de Gobierno y Políticas Públicas, Perú), Sapha Houssa (Alter Forum International), Mohamed Fadel Laatig (Alter Forum International). De igual manera, señalamos la participación de los alumnos del doctorado del departamento de Estudios Hispánicos: Fouziya Tabli, Imad Ouhaqi y Mohammed Ouahman; y de los estudiantes del máster América Latina: Hicham Idriss, Yassin Ouballi, Nabila Benslimane y Hayat Lagroum.

Señalamos el nombre de cada uno, en la medida que el trabajo de coordinación ha sido enorme. Cabe señalar que las gestiones logísticas, académicas, gubernamentales, gastronómicas, entre otras, han sido excelentes. Sin duda, todo el esfuerzo reunido en la persona de Bachir Edkhil, quien fue el anfitrión del evento y quien gestionó en patrocinio del Foro con entidades estatales como el ministerio encargado de los marroquíes residentes en el extranjero y los asuntos de la migración, el Ministerio de la Sociedad Civil y Relaciones con el Parlamento, el Ministerio de Información y Cultura, y la participación de Wilaya de la región de Laayoune, Tarfaya, Boujdour y Saguía El Hamra.

\section{Los idiomas en Marruecos}

En Marruecos, son oficiales los idiomas árabe y el amazigh, pero también se hablan otros dialectos/idiomas y dependiendo las zonas -de conquista- se mantienen hasta la actualidad. Por ejemplo, en el norte y en el sur del país se habla, además del árabe, español, mientras que en el centro del país se habla francés. No obstante, las cuestiones de la conquista han logrado expandir en todo el país el idioma francés como segunda lengua y -casi- ha desterrado al español. Adicionalmente, los árabes -digamos- de clase media, media alta y alta (profesionales y estudiantes universitarios), hablan varios idiomas: Árabe, Francés, Inglés y Español (aunque la minoría domina el último); algunos pocos conocen el idioma Italiano (producto de la migración también hacia Italia). Así, esta variedad lingüística evidencia que los ciudadanos marroquíes valoran de sobremanera romper fronteras idiomáticas con el objeto de entender al otro. En el Foro, participamos representantes de 11 países, donde cada uno expuso la ponencia en su idioma original. Afortunadamente contamos con traducción en simultáneo, lo que permitió que todos nos entendamos. Valoramos la buena disposición de los participantes -expositores y asistentes-, tanto docentes como alumnos, que nos ayudaron con la traducción al idioma requerido, especialmente en los momentos de pausa, almuerzos y cenas.

En cuanto a la pronunciación, en el idioma árabe, no se usa las vocales «e» $\mathrm{y}$ «o». Por ejemplo, cuando les decía que procedía del Perú, ellos no me entendían, sólo hasta que alguien me dijo que no pronuncian algunas vocales, entendí (claro, después de cuatro días). Por eso, ellos sí entienden la palabra «Pirú». Entonces, yo les decía que soy del «Pirú», a lo que me respondían: «ahhhhh, Machupicchu».

\section{Espiritualidad religiosa}

La mayoría de los marroquíes como todo el Magheb son musulmanes sunies $(99,4 \%)$, luego se distribuyen entre cristianos $(0,4 \%)$ y judíos $(0,1 \%)$. Los musulmanes rezan 5 veces al día, cada 24 horas. El llamado para las oraciones es muy particular: las campanas suenan durante los 5 momentos durante el día y la noche. Las horas varían, dependiendo del movimiento del sol. En líneas generales, las horas de oración fluctúan en los siguientes momentos: al alba (aproximadamente a las 4 a.m.), al medio día (entre la 1 y 2 p.m.), el atardecer (entre las 4 y 5 p.m.), cuando se esconde el sol (en promedio 9 o 10 p. m.), y por último, una hora después (a las 11 p.m.). La oración 
exige una predisposición de la persona: el cuerpo debe estar dispuesto con dirección a La Meca y se debe contar con un espacio adecuado, por ello, en todos los lugares públicos existen pequeños cuartos de adoración -o llamados espacios de culto- a los cuales se ingresa descalzo. Dentro, deben repetir rituales de limpieza para cada oración: lavado de manos (dos veces), lavado de brazos y codos, lavado del rostro y pies, pasar dos veces la manos limpias por sobre la cabeza, y solo entonces se está en disposición para la oración. Si no se cumple con este ritual, la oración no es válida. Es frecuente encontrar en los lugares de instituciones públicas los lavatorios como espacio de preparación para la oración. En las mezquitas (templos para la adoración) existen piletas para el lavado tanto para varones y mujeres, uno separado del otro. Estos lugares son también turísticos por ser monumentos históricos hermosos y muy grandes.

\section{Gastronomía árabe}

Si hay algo variado en el mundo árabe es su arte culinario. Color, sabor y abundancia caracterizan su gastronomía. Asimismo, el momento de consumir los alimentos tiene un sentido de comunidad, por eso las grandes fuentes de comida al centro de la mesa permiten que todos los comensales que se ubican alrededor puedan acercarse y tomar una porción del alimento. En la mesa siempre están a disposición pañuelos descartables, usados por todos para limpiarse las manos. Por lo general, primero se sirve una ensalada; luego, un pan grande y redondo que será disfrutado con el segundo plato -de fondo-, el cual puede ser algún guisado cuyos jugos serán consumidos con el pan; y para terminar, el postre, que consiste en una gran fuente con frutas de todas las variedades (de la región y algunas importadas). Acompañan el momento varios líquidos: agua y bebidas gaseosas (de las marcas mundialmente conocidas). No se consume licor, cuya ausencia es notoria para los europeos y latinoamericanos. A la salida de los lugares de almuerzos y cenas, existen lavatorios de manos (pedestal que contiene una tetera llena de agua limpia, jabón líquido y una toalla). El platillo nacional es el cuscús, similar a la quinua, el cual es el plato principal y que, acompañado de algunas carnes o mariscos, es la atracción de cualquiera. Durante las pausas se sirve café, el inconfundible té marroquí y bocadillos muy agradables: dulces de chocolate, pistachos, pastelillos, etc.

\section{Estilo de vida}

La vestimenta es muy característica de cada región. Los varones usan largas túnicas, cuyos bordados son distintivos de un caballero marroquí. La vestimenta de las mujeres tradicionales es muy colorida: normalmente lucen un tapado que envuelve su cuerpo desde la cabeza a los pies, y debajo suelen usar ropa de algodón que les ayuda a soportar las altísimas temperaturas del desierto. Además, dejan ver solo su rostro maquillado (el maquillaje de los ojos es muy llamativo); sin embargo, algunas pocas se cubren también el rostro, dejando solo los ojos al descubierto. Tanto hombres como mujeres, usan una especie de sandalias como calzado, cómodas y que les permite quitárselos en cualquier momento.

Con relación al género, notamos que todavía existe una fuerte tradición machista, no solo de parte de los hombres, sino también de las propias mujeres. Tuve la oportunidad de visitar una peluquería solo para mujeres, con mucho cuidado, protegiendo el cabello para que no lo vea algún caballero, porque solo los más íntimos de la familia pueden verlo: los hijos menores de edad y el esposo, nadie más. La peluquería se constituye como un espacio «liberado» para las mujeres, pues entre ellas sí existe una especie confianza, una correspondencia y respeto. Se lavan y arreglan el cabello, aunque luego se lo vuelvan a sujetar y tapar. Ellas saben que el cabello es su mayor atractivo femenino. 
Las diferencias por género se dan en muchísimos lugares, pero muchas mujeres marroquíes han logrado salir de esas barreras o vencerlas, volviéndose mucho más empoderadas. De hecho, participaron activamente de este evento. Además, se pudo visualizar todavía a algunas pocas conduciendo un vehículo. Estas actitudes evidencian la gran diferencia social, especialmente por género, pero que cada día se van superando.

\section{Desierto de Sahara}

Una parte del desierto está poblada. La otra parte corresponde al desierto llamado Sahara y es un lugar cautivante, de paz, sol y viento, y que está situado a solo 80 kilómetros del Caribe. La organización del evento preparó una noche en el desierto del Sahara. A una hora de la ciudad de Laayoune nos esperaba -quizás- lo más especial y diverso culturalmente. Fuimos a buscar a los camellos -para la foto de rigor- y ellos se asustaron con tantos turistas y buses por lo que se fueron; luego ya era muy tarde y debían descansar. Las carpas, las bellas alfombras -todos descalzos-, la bienvenida con un vaso con leche de camello y dátiles (fruta seca de la región) eran solo una parte de la velada. Cuando creímos que volveríamos a la ciudad, nos invitaron a otra carpa para la cena -ya con zapatos- con platillos tradicionales: dulces, ensaladas, pan, cuscús, langosta y fruta. El fin de fiesta, con música y baile incluido, contó con la participación de los extranjeros -no sabíamos bien qué hacíamos, pero disfrutamos el momento-. Las palabras de agradecimiento de y para los extranjeros, participación de autoridades marroquíes, los obsequios y los diplomas, dieron por concluida la experiencia de observación participante más espectacular que alguien que va desde el Perú se pueda imaginar.

\section{Mi paso por Rabat y Casablanca}

Rabat es la capital cultural y de gobierno del Reino de Marruecos. El sociólogo colombiano Gabriel Retrepo nos invitó a participar de la presentación de un libro Marruecos, Rosa de los vientos, en el Instituto Cervantes de Rabat, como parte de un evento organizado por la Embajada de Colombia, que a su vez, cumplía 20 años de relaciones internacionales con Marruecos. El evento, de corte académico literario, contó además con la participación del escritor colombiano, Luis Fayat, quien presentó una nueva edición de su libro Los parientes de Esther después de 20 años de haber sido publicado. Asistieron representantes de diferentes embajadas, especialmente de Iberoamérica. También participaron docentes y estudiantes de la Universidad Mohamed V de Rabat, con quienes tuve oportunidad de intercambiar las experiencias de trabajo que tuvimos con personas de diversas nacionalidades. Previa coordinación, el embajador del Perú, Sr. Arturo Chipoco Cáceda, el Ministro Consejero, Sr. Carlos Cueto, y yo, tuvimos la oportunidad de conocernos en la misma reunión del Instituto Cervantes, donde conversamos sobre la relevancia de la invitación marroquí a una profesora peruana de una universidad pública tan importante como lo es la Universidad Nacional Mayor de San Marcos, conocida internacionalmente.

Al día siguiente, las relaciones internacionales tuvieron lugar. Temprano, Gabriel Restrepo, Luis Fayat y su esposa Rosario, y yo, fuimos invitados a la embajada de España, donde nos recibió el embajador Sr. Ricardo Díez Hochleitner, y tuvimos la oportunidad de comentarle las actividades que hemos realizado en el país. Fue una muy agradable reunión. Hacia el mediodía, nos esperaba la embajadora de Colombia, Sra. María del Pilar Gómez Valderrama, quien nos agasajó con bocadillos de la región y compartimos, también, nuestras actividades. Por la tarde, a partir de las dos de la tarde, como teníamos mucho de qué hablar con el embajador peruano, fui invitada a visitar la embajada (local propio, ubicada en una pequeña plaza que lleva por nombre Perú) y, luego, a almorzar a la casa del embajador. Una tarde simpática, con conversaciones sobre la universidad en el Perú, los problemas nacionales, los peruanos migrantes, 
y el trabajo diplomático y académico. Más tarde (la luz del día está presente hasta después de las nueve de la noche), conocí algo de la ciudad: el mausoleo de Mohamed V, la Torre de Hassan, el barrio de Kasbah de Udayas -callecitas pintadas de azul y blanco-, altos edificios (antiguos) con sus hermosos Jardines Andaluces de Rabat, y el mirador al Océano Atlántico. Hermosos lugares. Al final de la jornada, gracias a la fina cortesía de la embajada peruana me trasladaron a la ciudad de Casablanca, situado a una hora -en auto- de Rabat.

Casablanca es la ciudad más importante de Marruecos y es considerada como la capital comercial y financiera. Me quedé hasta el día siguiente en la ciudad debido a que es el aeropuerto internacional más importante del continente africano y debía tomar el vuelo de retorno a casa (vía Madrid). Esta ciudad es absolutamente diferente, ya que se ve mucho movimiento, turismo, y áreas en reconstrucción (lo que indica que hay dinámica monetaria). En un lugar enorme como este, en 1984, se inició la construcción de un templo gigante, llamado Hasann Du (Mezquita), cuyo trabajo se terminó después de 10 años. La torre principal mide 220 metros de altura, el diseño es el típico marroquí con algunas aplicaciones europeas, su capacidad es de 25 mil personas al interior del templo), la disposición del cuerpo de los que oran debe ser hacia la Meca, y cuenta con diversas salas para las ceremonias, lavatorios y un gran techo de madera desplegable para contemplar el cielo. También cuenta con una explanada exterior, la cual, en la época del Ramadán (mes de ayuno para los musulmanes) recibe a 80 mil personas. Todo el conjunto es parte de la expresión cultural religiosa más interesante antes conocida.

En suma, la visita a Marruecos ha sido una grata experiencia en la que valoramos la organización de evento y el cuidado para con los invitados, la amabilidad y acogida de su gente, la variedad gastronómica, la manera protocolar de recibir a los invitados, la recepción en el desierto, el manejo de diversos idiomas, y la música tradicional. Todo ello hizo que nuestro paso por un país tan bonito e interesante, entorno de la academia, sea la experiencia social más interesante. 


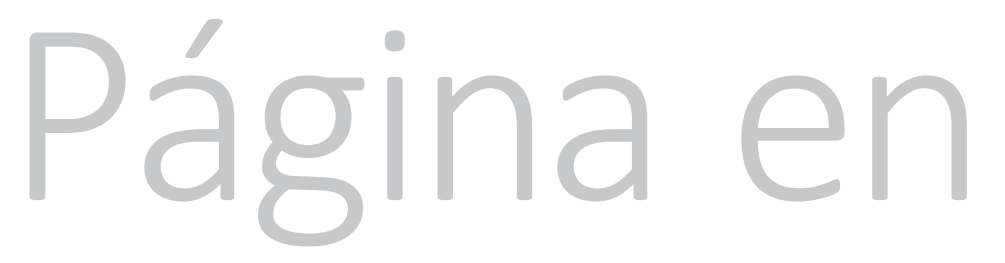

blanco 